\title{
OPTIMIZED MANAGEMENT OF TRANSFUSION IN A PEDIATRIC SURGICAL BLOCK
}

Pérez Ferrer A., Viejo Llorente A., Latorre J.

\section{Background:}

La Paz University Hospital, Dept of Anaesthesiology \& Intensive Care, Madrid, Spain

- Tolerance to hypovolemia is physiologically lower in the pediatric patient

- Due to their lower blood volume, bleeding volumes of minor importance in an adult, can be critical in the child.

- It is not uncommon to request blood components from the operating room in surgical procedures in which it is not certain that it will be transfused but, if necessary, will require it immediately.

- Frequently these blood components have to be discarded in case of non-transfusion.

AlM:

To analyze the impact of a new transport and storage protocol of blood components in portable coolers (PC), between the Blood Bank (BB) and the operating room.

\section{Method:}

- An analysis of the number of blood components returned to the BB from the operating room over a period of 7 years (2009-2015) was carried out.

- We also analyze the economic cost of the discarded packages.

During the first 7 months, the impact of the implementation of a protocol for the delivery of blood components to the operating room in PC with controlled temperature was evaluated, both in the number of blood components that were reused and the savings that this entailed.

\section{Results and Discussion:}

- Discarded units in the previous 7 years were:

\begin{tabular}{c|c|c|c|c|c|c|c|} 
& 2009 & 2010 & 2011 & 2012 & 2013 & 2014 & 2015 \\
\hline Units & 277 & 163 & 146 & 108 & 129 & 110 & 110 \\
\hline
\end{tabular}
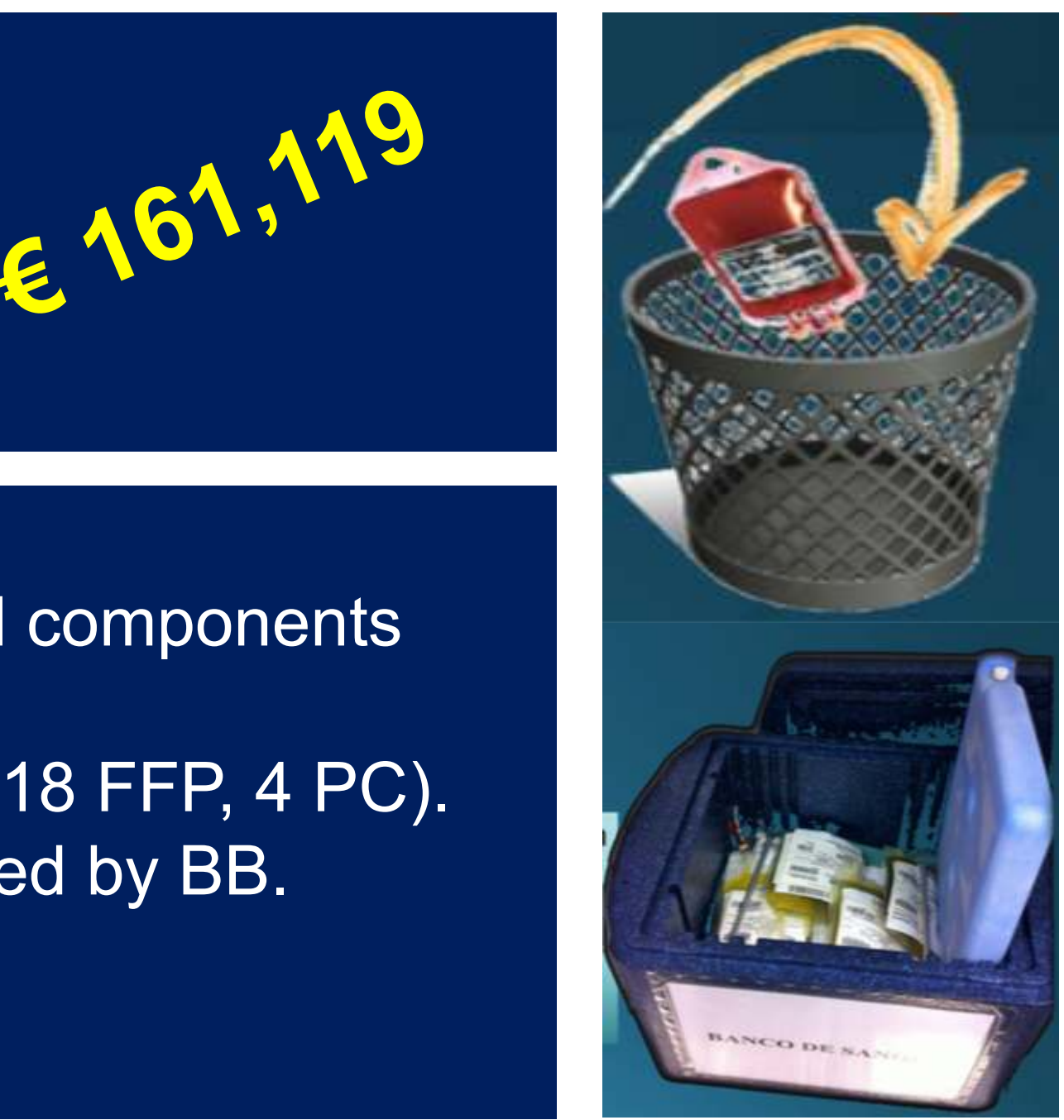

- On the first 7 months :

$\checkmark 108$ PC were sent, with a total of 405 Units of blood components (243 RBC, 146 FFP and 16 PC).

$\checkmark 74$ units were returned to BB and reused (52 RBC, 18 FFP, 4 PC).

$\checkmark$ Only 2 units of RBC and 2 of FFP have been rejected by BB.

- The savings calculated was $€ \mathbf{8 , 7 0 0}$.

Conclusions:

* The Transfusion committee detected an easily avoidable expense through the buy of 3 PCs, accumulators and temperature control systems, which were amortized in a single month.

* The development of a protocol for sending PC with blood components to the children's surgical block will allow an estimated annual savings of $€ 15,000$. 